\title{
RESOURCE RESERVATION SCHEMES FOR OPTICAL BURST SWITCHING NETWORKS
}

Since the current optical communication networks are based on internet protocol and demands for transmission capacity and speed are growing, the optical burst switching presents the solution for future high-speed wavelength division multiplexing optical networks. In order to successfully transmit bursts over optical burst switching network, resource reservation schemes have to be implemented. According to the way of resource reservation, reservation schemes use one-way reservation or two-way reservation. The schemes with one-way reservation are used mainly for optical burst switching networks. The survey of one-way resource reservation schemes is presented. The basic operations and properties of one-way resource reservation schemes are described.

Keywords: Optical burst switching, resource reservation scheme, one-way reservation.

\section{Introduction}

With growing demands of Internet Protocol (IP) services for transmission capacity and speed, the Optical Burst Switching (OBS) presents the solution for future high-speed Wavelength Division Multiplexing (WDM) optical networks. OBS is a technology for transmitting large amounts of data bursts through a transparent optical switching network. OBS combines the best elements of optical packet switching and wavelength routing, but avoids their shortcomings [1].

An OBS network consists of the edge nodes and the core nodes interconnected with each other with WDM links (Fig. 1). Two types of edge nodes are differentiated in OBS network, the ingress (core) and the egress (destination) edge nodes that are based on interface of the classical IP network and the OBS network [1-3].

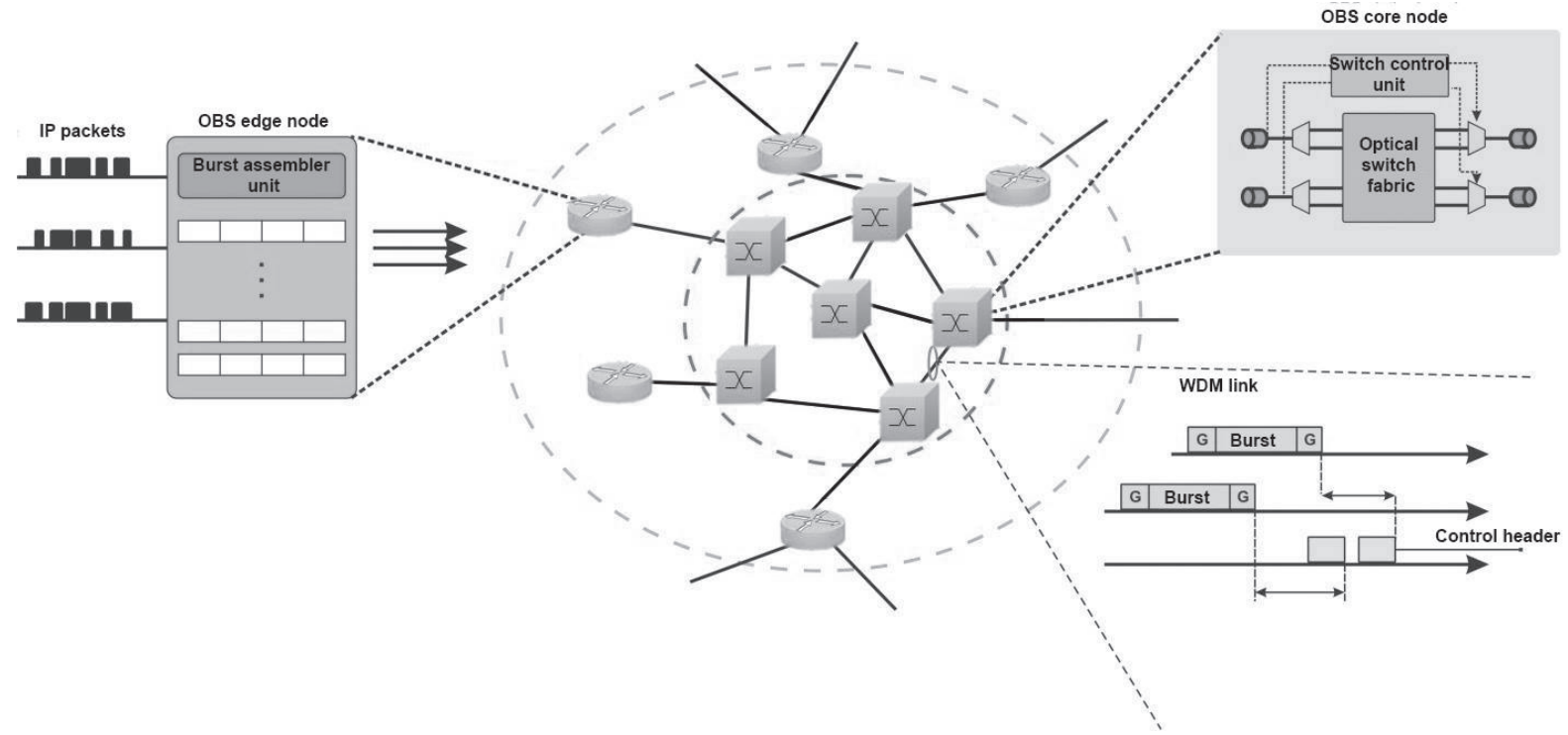

Fig. 1 The architecture of OBS network

\footnotetext{
* Michaela Solanska, Miroslav Markovic, Milan Dado

Department of Telecommunications and Multimedia, Faculty of Electrical Engineering, University of Zilina, Slovakia

E-mail: michaela.solanska@fel.uniza.sk
} 
Ingress edge nodes electronically assembly several incoming IP packets with the same destination into constant or variable length optical bursts, which stay in optical domain until they arrive to the egress edge node. To each optical burst is generated header, necessary to correct the switching structure setting in every core node, through which the burst will be passing. Just before the burst is transported, the control header is sent, to inform all the core nodes along the road about the burst arrival. The control header is sent through the independent channel, which is on different wavelength as transferred optical burst (out-of-band signaling). In each core node, the control header is converted by optical-electronic-optical $(\mathrm{O} / \mathrm{E} / \mathrm{O})$ conversion, due to not available all optical control circuits. On the basis of an information contained in the control header is made the setting of switching structure in core node, which is processed in control circuit. The information contains the time delay between control header and optical burst (referred to as offset), size of the optical burst, required output port, incoming wavelength. Then the new header for transmitting burst is generated. This header is again sent from the core node in advance of the optical burst. This repeats until the optical burst does not arrive to the egress edge node. The purpose of the egress edge node is to disassembly the optical burst in a condition that has been set before entering into the edge node [1-9].

\section{One-Way Resource Reservation Schemes}

The main issue in OBS network is the random collision and burst loss. To successfully transmit bursts over OBS network and reach destination node, resource reservation schemes have to be implemented to allocate resources and configure optical switches for that burst at each node. According to the process of resource reservation, resource reservation schemes may be classified into two main classes: one-way reservation and two-way reservation. OBS networks use mainly reservation schemes with one-way reservation. By one-way reservation bursts are sent shortly after the control header and the source node does not wait for response about the success of the reservation of a full path sent by the destination node. A number of one-way resource reservation schemes have been proposed for OBS, including Tell-And-Go (TAG), Just-In-Time (JIT), Enhanced Just-In-Time (E-JIT), Enhanced Just-In-Time Plus (E-JIT ${ }^{+}$), Just-In-Time Plus (JIT ${ }^{+}$), Just-Enough-Time (JET), Horizon, JumpStart [4-9].

In the Tell-And-Go (TAG) reservation scheme a source node sends a control header that contains Setup message. Immediately after control header source node sends a data burst. This reservation scheme is rather idealistic and non-realistic, due to the lack of time of the receiving node to process the Setup message and to configure its switch structure, so it can switch the incoming burst to required output port. To apply these scheme to the real life, it is necessary to configure the node in advance to switch the burst, or equip it with optical buffers, which delay the bursts, while the node will process the Setup message and configures its switching structure. TAG is practical only if the switch processing time of the Setup message and the optical switch configuration time are very short. Therefore, due to the critical limitation of TAG the study is focused on aforementioned one-way resource reservation schemes [4-9].

Just-In-Time (JIT) reservation scheme was proposed by Wei and McFarland in [4]. JIT is resource reservation scheme with immediate reservation and it uses explicit releases to set free the switching structure resources. JIT operates as follows: an output wavelength is reserved for a burst immediately after the arrival of the corresponding Setup message. If a wavelength cannot be reserved immediately, then the Setup message is rejected and the corresponding burst is dropped (Fig. 2). JIT uses first-come, first- served (FCFS) service on each wavelength, because bursts are scheduled in the order in which their Setup messages arrive at the node [4-9].

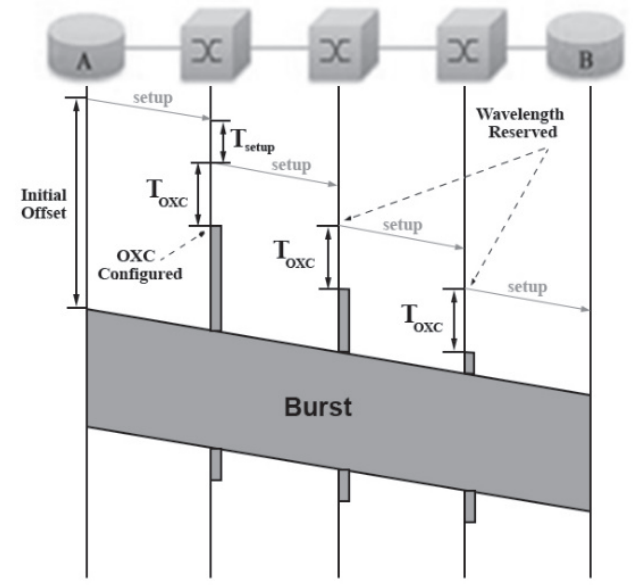

Fig. 2 Schematic representation of JIT reservation scheme [5]

Just-In-Time Plus $\left(\mathrm{JIT}^{+}\right)$reservation scheme was proposed by Teng and Rouskas in [5]. $\mathrm{JIT}^{+}$is resource reservation scheme with immediate reservation and it uses estimated releases to set free the switching structure resources. $\mathrm{JIT}^{+}$is a modified and improved version of JIT reservation scheme. $\mathrm{JIT}^{+}$combines simplicity of JIT with the utilization of the time horizon that is used by delayed resource reservation schemes. JIT $^{+}$adds limited burst scheduling (for a maximum of two bursts per channel). $\mathrm{JIT}^{+}$operates as follows: an output wavelength is reserved for a burst only if (1) the arrival time of the burst is later than the time horizon defined for the wavelength and (2) the wavelength has at most one other reservation. JIT $^{+}$uses FCFS service on each wavelength [4-9].

Enhanced Just-In-Time (E-JIT) reservation scheme was proposed by Rodrigues, Freire and Monteiro in [7]. E-JIT is resource reservation scheme with immediate reservation and it uses estimated (or implicit) releases to set free the switching 
structure resources, as the Setup message also carries the information of burst length and burst offset length, allowing each node to estimate the latest time the resources are no longer assigned to a burst transmission. E-JIT improves and optimizes JIT reservation scheme, keeping all the advantages of its simplicity in terms of implementation. Optimization is achieved by the improvement of data channel scheduling. The period of time in which wavelength remains in reserved status is reduced, so that it results in the optimization of the wavelength utilization and potentially reduce the burst loss probability. E-JIT operates as follows: an output wavelength is reserved for a burst immediately after the arrival of the corresponding Setup message, if (1) this wavelength is free or (2) if it is reserved, the end time of the last switched burst is smaller than the actual time to process the Setup message. If a wavelength cannot be reserved immediately, then the Setup message is rejected and the corresponding burst is dropped. E-JIT uses FCFS service on each wavelength [4-9].

Enhanced Just-In-Time Plus (E-JIT ${ }^{+}$) reservation scheme was proposed by Rodrigues, Gregorio and Vasilakos in [9]. E-JIT ${ }^{+}$ is resource reservation scheme with immediate reservation and it uses estimated (or implicit) releases to set free the switching structure resources. E-JIT ${ }^{+}$is based on $\mathrm{JIT}^{+}$reservation scheme, integrates functions of E-JIT reservation scheme in terms of scheme operations, and intends to increase the performance of OBS networks, maintaining the simplicity of JIT reservation scheme in terms of implementation. E-JIT+ uses the time horizon concept, which determines whether a channel is ready to accept new bursts (in order to make possible the calculation of the time horizon value, Setup messages carry information with the burst offset and the burst length values). E-JIT ${ }^{+}$operates as follows: an output wavelength is reserved for a burst if the arrival time of the burst occurs later than the time horizon of that wavelength. Otherwise, the Setup message is rejected and the corresponding data burst is dropped. E-JIT ${ }^{+}$uses FCFS service on each wavelength [4-9].

Just-Enough-Time (JET) reservation scheme was proposed in [10-12]. JET is the best known reservation scheme with delayed reservation that uses void filling. JET uses estimated releases to set free the switching structure resources. JET operates as follows: an output wavelength is reserved for a burst if the arrival time of the burst (1) is later than the time horizon defined for the wavelength, or (2) coincides with avoid on the wavelength, and the end of the burst (plus the configuration time of optical crossconnect) is sooner than the end of the void. If, after arrival of the Setup message, it is determined that none of these conditions are satisfied for any wavelength, then the Setup message is rejected and the corresponding burst is dropped (Fig. 3). JET uses non FCFS service on each wavelength [8, 10, 11 and 12].

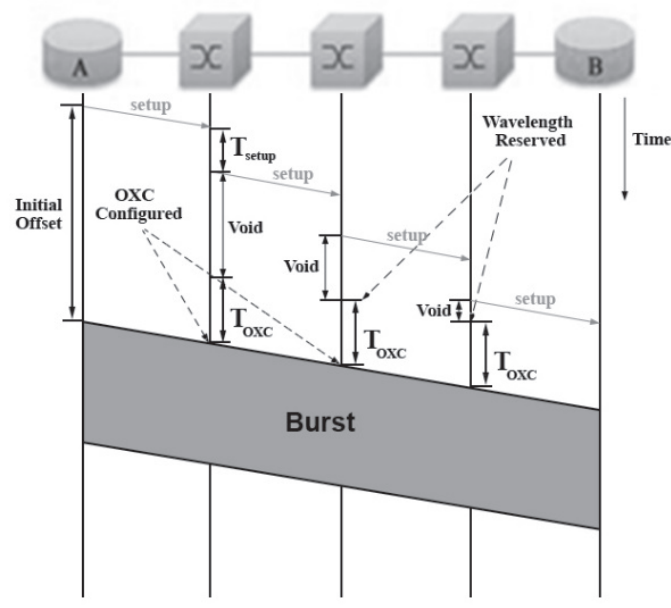

Fig. 3 Schematic representation of JET reservation scheme [5]

Horizon reservation scheme was proposed by Turner in [13]. Horizon is resource reservation scheme with delayed reservation that does not perform any void filling. Horizon scheme takes its name from the fact that each wavelength is associated with a time horizon for burst reservation purposes. This time horizon is defined as "the earliest time after which there is no planned use of the wavelength". Horizon operates as follows: an output wavelength is reserved for a burst only if the arrival time of the burst is later than the time horizon of the wavelength. If, after arrival of the Setup message, it is determined that the arrival time of the burst is earlier than smallest time horizon of any wavelength, then the Setup message is rejected and the burst corresponding burst is dropped. Horizon uses FCFS service on each wavelength [6, 8 and 13].

JumpStart is a joint project supported by Advanced Research and Development Agency (ARDA) developed by the North Carolina State University (NCSU) and MCNC Research and Development Institute [6]. JumpStart reservation scheme uses immediate and delayed reservation. JumpStart combines control and signalization simplicity together with multiple functions, including support for multicast and different types of connection. JumpStart operates as follows: a source node first sends the Setup message to core node with information related to the burst transmission, including the source and destination addresses. If the core node can switch the burst, it returns the Setup ACK message to the source node. Moreover, it forwards the Setup message to the next node. Otherwise, the core node refuses the Setup message and returns a reject message to the source node and the corresponding burst is dropped. In this case, the source node enters in an idle period waiting for another burst. When a new burst arrives, the edge node repeats the process $[6,14,15$ and 16]. 
Main properties of reservation schemes

\begin{tabular}{ccccc}
\hline \multirow{2}{*}{ Reservation scheme } & Reservation process & $\begin{array}{c}\text { The release process of reservated } \\
\text { resources }\end{array}$ & Burst service & $\mathbf{T}_{\text {Setup }}$ \\
\hline JIT & Immediate & Explicit & FCFS & $12,5 \mu \mathrm{s}$ \\
\hline JET & Delayed & Estimate & Non FCFS & $50 \mu \mathrm{s}$ \\
\hline E-JIT & Immediate & Estimate & FCFS & $12,5 \mu \mathrm{s}$ \\
\hline E-JIT $^{+}$ & Immediate & Estimate & FCFS & $12,5 \mu \mathrm{s}$ \\
\hline JIT $^{+}$ & Immediate & Estimate & FCFS & $12,5 \mu \mathrm{s}$ \\
\hline Horizon & Delayed & - & FCFS & $25 \mu \mathrm{s}$ \\
\hline JumpStart & Immediate/Delayed & - & - & $12,5 \mu \mathrm{s}$ \\
\hline
\end{tabular}

The basic features of reservation schemes are summarized in Table $1\left(\mathrm{~T}_{\text {Setup }}\right.$ is the amount of time it takes an OBS node to process the Setup message under reservation scheme).

\section{Comparison of reservation schemes}

The basic reservation schemes JET, JIT, Horizon are compared in terms of burst loss probability that is the main issue in OBS networks. The analytical burst loss probability of reservation schemes was calculated on the basis of study in [5]. Figure 4 shows the burst loss probability as a function of number of wavelengths (data channels) per optical fiber in a single node for reservation schemes. The burst loss probability decreases with

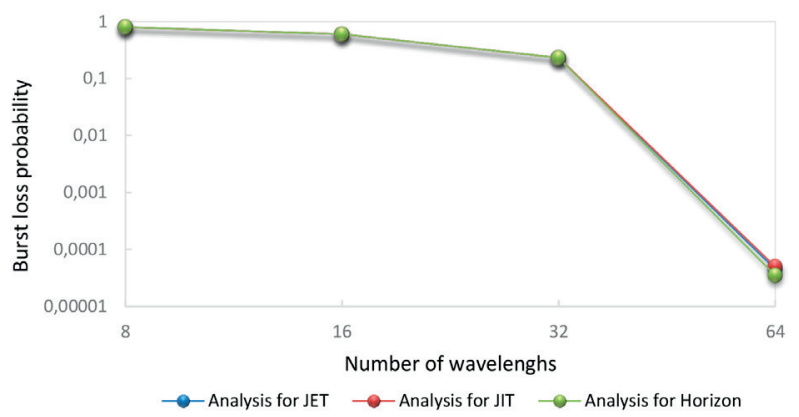

Fig. 4 Burst loss probability as a function of number of wavelengths per optical fiber in a single node for JET, JIT and Horizon reservation schemes then increasing number of wavelengths and the performance of each reservation schemes is very similar.

\section{Conclusion}

The reservation schemes play an important role for data transmission. The reservation schemes allocate resources along path from source to destination node. The one-way resources reservation schemes for OBS networks, the basic operations and properties of reservation schemes are described. The next goal is to compare analytical and simulation burst loss probability of JET, JIT and Horizon reservation schemes based on the OBS network model that was created using $\mathrm{OMNeT}++$ simulation environment. To study the impact of the number of data channels, number of hops, number of nodes, traffic load, topology selection of OBS network on the performance of each reservation scheme.

\section{Acknowledgements}

This work is supported by the Slovak Research and Development Agency under the project APVV-0025-12 ("Mitigation of stochastic effects in high-bitrate all-optical networks") and the European Regional Development Fund and the Slovak state budget for the project "Research Centre of University of Zilina”, ITMS 26220220183.

\section{References}

[1] PAPADIMITRIOU, G., PAPAZOGlOU, CH., POMPORTSIS, A.: Optical Switching, Hoboken : Wiley, 2007.

[2] UMALE, M. R., JADHAV, S. S., NARAVADKAR, S. D.: Optical Burst Switching (OBS) for Next Generation Optical Transport Networks, Emerging Trends in Computer Science and Information Technology, ETCSIT, 2011.

[3] SALEH, A. A. M., SIMMONS, J. M.: All-Optical Networking-Evolution, Benefits, Challenges, and Future Vision, IEEE J. \& Magazines, vol. 100, No. 5, 2012.

[4] WEI, J. Y., MCFARLAND, R. I.: Just-In-Time Signaling for WDM Optical Burst Switching Networks, J. of Lightwave Technology, vol. 18, No. 12, 2019-2037, 2000.

[5] TENG, J., ROUSKAS, G. N.: A Detailed Analyses and Performance Comparison of Wavelength Reservation Schemes for Optical Burst Switched Networks, Photonic Network Communications, vol. 9, No. 3, 311-335, 2005. 
[6] RODRIGUES, J. J. P. C., FREIRE, M. M., LORENZ, P.: One-Way Resource Reservation Protocols for IP over Optical Burst Switches Mesh Networks, Systems Communications, ICW'05, 229-234, 2005.

[7] RODRIGUES, J. J. P. C., FREIRE, M. M., GARCIA, N. M., MONTEIRO, P. M. N. P.: Enhanced Just-In-Time: A New Resource Reservation Protocol for Optical Burst Switching Networks, $12^{\text {th }}$ IEEE Symposium on Computers and Communications, ISCC 2007, 121-126, 2007.

[8] RODRIGUES, J. J. P. C., VAIDYA, B.: Evaluation of Resource Reservation Protocols for IP over OBS Networks, $11^{\text {th }}$ Intern. Conference on Transparent Optical Networks, ICTON'09, 1-4, 2009.

[9] RODRIGUES, J. J. P. C., GREGORIO, J. M. B., VASILAKOS A. V.: Enhanced Just-In-Time Plus Protocol for Optical Burst Switching Networks, J. of Optical Engineering, vol. 49, No. 7, 2010.

[10] QIAO, C.: Optical Burst Switching - A Novel Paradigm, Optical Internet Workshop, 1997.

[11] YOO, M., QIAO, C.: Just-Enough-Time (JET): A High Speed Protocol for Bursty Traffic in Optical Networks, IEEE/LEOS Conference on Technologies for a Global Information Infrastructure, 26-27, 1997.

[12] QIAO, C., YOO, M.: Optical Burst Switching (OBS) - A New Paradigm for an Optical Internet, J. of High Speed Networks, vol. 8, No. 1, 69-84, 1999.

[13] TURNER, J. S.: Terabit Burst Switching, J. of High Speed Networks, vol. 8, No. 1, 3-16, 1999.

[14] BALDINE, I., ROUSKAS, G. N., PERROS, H. G., STEVENSON, D.: JumpStart: A Just-In-Time Signaling Architecture for WDM Burst-Switched Networks, IEEE Communications Magazine, 82-89, 2002.

[15] ZAIM, A. H., BALDINE, I., CASSADA, M., ROUSKAS, G. N., PERROS, H. G., STEVENSON, D.: The JumpStart Just- In-Time Signaling Protocol: A Formal Description Using EFSM, Optical Engineering, vol. 42, No. 2, 568-585, 2003.

[16] BALDine, I., ROUSKAS, G. N., PERROS, H. G., STEVENSON, D.: Signaling Support for Multicast and QoS within the JumpStart WDM Burst Switching Architecture, Optical Networks Magazine, vol. 4, No. 6, 68-80, 2003. 